\title{
The Theory of 2-Structures
}

\section{A Framework for Decomposition and Transformation of Graphs}


Addresses:

\section{Professor Andrzej Ehrenfeucht}

Department of Computer Science

University of Colorado at Boulder

Boulder, Co 80309, U.S.A.

Corresponding author:

Dr. Tero Harju

Department of Mathematics

University of Turku

FIN-20014 Turku, Finland

E-mail: harju@utu.fi

\section{Professor Grzegorz Rozenberg}

Leiden Institute for Advanced Computer Science

Leiden University

P.O. Box 9512, 2300 RA Leiden, The Netherlands 


\title{
The Theory of 2-Structures
}

\section{A Framework for Decomposition and Transformation of Graphs}

\author{
A. Ehrenfeucht \\ University of Colorado, Boulder, USA \\ T. Harju \\ University of Turku, Finland \\ G. Rozenberg \\ Leiden University, The Netherlands
}




\section{Published by}

World Scientific Publishing Co. Pte. Ltd.

P O Box 128, Farrer Road, Singapore 912805

USA office: Suite 1B, 1060 Main Street, River Edge, NJ 07661

UK office: 57 Shelton Street, Covent Garden, London WC2H 9HE

\section{British Library Cataloguing-in-Publication Data}

A catalogue record for this book is available from the British Library.

\section{THE THEORY OF 2-STRUCTURES}

Copyright $\odot 1999$ by World Scientific Publishing Co. Pte. Ltd.

All rights reserved. This book, or parts thereof, may not be reproduced in any form or by any means, electronic or mechanical, including photocopying, recording or any information storage and retrieval system now known or to be invented, without written permission from the Publisher.

For photocopying of material in this volume, please pay a copying fee through the Copyright Clearance Center, Inc., 222 Rosewood Drive, Danvers, MA 01923, USA. In this case permission to photocopy is not required from the publisher.

ISBN $981-02-4042-2$

Printed in Singapore by Regal Press (S) Pte. Ltd. 
to Pat, Eija and Maja 
This page is intentionally left blank 


\section{Preface}

The aim of this book is to give a self-contained treatment of the theory of 2-structures, which provides a convenient framework for decomposition and transformation of mathematical systems, where one or several different binary relationships hold between the objects of a system.

The 2-structures are intimately related to edge coloured directed graphs, and, indeed, in the second half of the book, we shall study labelled 2-structures that can be identified with these directed graphs. We use the generic name '2-structure' in order to emphasize that we investigate systems of binary relations in an abstract setting.

Various methods to decompose graphs and other related mathematical structures related to them have been studied in the literature. Decompositions of combinatorial and algebraic structures are special cases of the divide-and-conquer method, where a large problem is partitioned into smaller subproblems, and a method is given to link solutions of the subproblems into a solution of the original one. The clan decomposition of 2-structures, also known as the modular decomposition or the substitution decomposition, is an example of such a decomposition - it is closely related to the decomposition by quotients in algebra.

This book covers two main topics.

- The 'static' part of the theory of 2-structures (Chapters 3 through 10 ) is concerned mainly with the decomposability and indecomposability (or primitivity). In Chapters 3 through 7 we consider 'unlabelled' 2 -structures that are defined by an equivalence relation on the edges. The key notion here is that of a clan of a 2-structure $g$, which is a subset $X$ of nodes such that an element $y \notin X$ cannot 
make a distinction between the elements of $X$ by using the equivalence relation provided by $g$. To make the classification of the edges more specific one adds a function labelling the equivalence classes of edges, obtaining in this way a labelled 2-structures; these structures are studied in Chapters 8 through 10.

- The 'dynamic' part of the theory (Chapters 11 through 15) is concerned with the local transformations (or switchings) of the 2-structures. Here the set of labels of the edges is given a group structure. In this way one obtains switching classes of labelled 2-structures, where a switching class consists of 2-structures that can be transformed into each other, and one uses the group operations, applied locally in the nodes, to induce transformations of the labels of the edges.

Much of the motivation for the research presented in this book comes from the area of graph grammars, where one considers the global effect of local transformations defined through grammatical productions. We believe that (especially the dynamic part of) the theory of 2-structures enriches the study of graph grammars by making available the tools from group theory and the theory of switching.

At the end of each chapter we provide notes on the references with annotations that we feel are relevant for the chapter.

We have also included exercises, many of which are straightforward observations that provide an opportunity for the reader to check her/his understanding of the discussed material. Some of the exercises are more demanding, especially those that give a result from a research article. The references for these exercises are also given in the notes on references.

Chapter 1 introduces the basic notions such as partial orders, semigroups, monoids, and groups. Groups are essential from Chapter 11 onwards, but they are used earlier than that in the form of automorphism groups.

Chapter 2 gives, in a compact way, the graph theoretical preliminaries.

Chapter 3 introduces the 2-structures, their clans and factors. The closure properties of the clans are investigated. Also, prime clans are introduced and discussed - they will be crucial in the decompositions of the 2-structures.

Chapter 4 continues the discussion of the basic notions of 2-structures, but now from a more algebraic point of view. In this chapter we study 
the operations of quotients and homomorphisms, and their relationships to clans.

Chapter 5 makes the use of the results from Chapters 3 and 4 to prove the clan decomposition theorem. We also show that each 2-structure $g$ has a very special 'construction tree', called the shape of $g$. It represents the 'canonical' construction of $g$.

Chapter 6 is devoted to primitive 2-structures - those 2-structures that do not have nontrivial clans, and thus are indecomposable.

Chapter 7 considers a subclass of 2-structures, called angular 2-structures, that results by forbidding primitivity on the lowest possible level. These 2-structures are interesting on their own, and have applications in partially ordered structures.

Chapter 8 starts our journey to local transformations by modifying some of the results of the previous chapters to labelled 2-structures. The clan decomposition theorem is given another proof in this chapter.

Chapter 9 considers a special topic of automorphisms of labelled 2-structures. Automorphisms are important in all algebraic and combinatorial systems, since they measure the 'inner symmetry' of the system.

Chapter 10 discusses primitivity from the point of view of the labelled 2-structures.

Chapter 11 considers switching of (directed) graphs. At the same time it is an introduction to the theory of switching of 2-structures. This chapter, as well as the following chapters, owe much of their motivation and results to switching of (undirected) graphs - also known as Seidel switching.

Chapter 12 defines the switching classes of the labelled 2-structures that take their edge labels from a group. This chapter also gives an interpretation of the switching from the point of view of local transformations of networks.

Chapter 13 provides tools to study the switching classes. Also, we count here the number of the elements in switching classes in the case where the group of labels is finite.

Chapter 14 considers the quotients for switching classes. Surprisingly enough, also the shape has a counterpart for the switching classes.

Chapter 15 studies the properties of labelled 2-structures that remain invariant in a switching class, that is, those properties that remain unchanged under the local transformations of the 2-structures. 
The authors are grateful to their friends and colleagues, who have cooperated in the development of the theory of 2-structures. Acknowledgements are due to Joost Engelfriet, Jurriaan Hage, Henrik Jan Hoogeboom, Ross McConnell, Paulien ten Pas and Andrzej Proskurowski.

We thank Rudy van Vliet for his comments on the first version of the manuscript of this book.

The authors are indebted to Training and Mobility of Researchers Network General Theory of Graph Transformation Systems (GETGRATS) for supporting the collaboration on this book.

A.E., T.H. and G.R.

December 1, 1998 


\section{Contents}

Preface vii

Chapter 1 Preliminaries 1

1.1 Notations . . . . . . . . . . . . . . . . 1

1.1.1 Sets and functions . . . . . . . . . . . . 1

1.1 .2 Closure operators . . . . . . . . . . . . . 3

1.1.3 Relations . . . . . . . . . . . . . . 4 4

1.1.4 Equivalence relations . . . . . . . . . . . 6

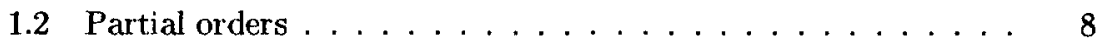

1.2.1 Downsets . . . . . . . . . . . . . 8

1.2 .2 Order embeddings $\ldots \ldots \ldots \ldots \ldots$

1.2 .3 Linear orders . . . . . . . . . . . . . . . . . 10

1.3 Semigroups and groups $\ldots \ldots \ldots \ldots \ldots \ldots$

1.3.1 Notations for semigroups and monoids . . . . . . . . 11

1.3.2 Free monoids (with involution) . . . . . . . 12

1.3.3 Preliminaries on groups . . . . . . . . . . . . . 14

1.3.4 Group actions ................ 16

1.3.5 Free groups, commutators and verbal identities . . . . 17

Chapter 2 Graph Theoretical Preliminaries 21

2.1 Directed and Undirected Graphs . . . . . . . . . . . . 21

2.1.1 Basic notions . . . . . . . . . . . . . . . . 21

2.1 .2 Connectivity of graphs . . . . . . . . . . . . . 24

2.1.3 Some special graphs . . . . . . . . . . . . . . . 25

2.2 Comparability graphs . . . . . . . . . . . 27 
2.2.1 Transitively oriented graphs . . . . . . . . . . 27

2.2.2 Permutation graphs and cographs . . . . . . . . . 29

2.2.3 Construction trees of cographs . . . . . . . . . 31

Chapter $3 \quad$ 2-Structures and Their Clans 35

3.1 Introduction and representations . . . . . . . . . 35

3.1.1 Definition of a 2-structure . . . . . . . . . . 35

3.1 .2 Isomorphic 2 -structures $\ldots \ldots \ldots . \ldots . \ldots . \ldots 37$

3.1.3 Reversibility . . . . . . . . . . . . 38

3.2 Substructures and clans $\ldots \ldots \ldots \ldots . \ldots . \ldots . \ldots$

3.2.1 Substructures, clans and factors . . . . . . . . 39

3.2.2 Refinements and similarity . . . . . . . . . 41

3.2 .3 Reversible version . . . . . . . . . . . . . . 42

3.2.4 Graphs and packed components . . . . . . . . . . 43

3.2.5 Some special 2-structures . . . . . . . . . . . 46

3.3 Closure properties of clans . . . . . . . . . . . . . . 47

3.3.1 Basic closures . . . . . . . . . . . . . . . 47

3.3.2 Sibas: set theoretic closure properties . . . . . . . . 49

3.3.3 Clans of factors . . . . . . . . . . . . . . 51

3.4 Prime clans . . . . . . . . . . . . . . . . 52

3.4 .1 Prime members in sibas . . . . . . . . . . 52

3.4 .2 Minimal overlapping clans . . . . . . . . . . . 53

Chapter 4 Quotients and Homomorphisms $\quad 57$

4.1 Quotients ....................... 57

4.1.1 Factorizations and quotients $\ldots \ldots \ldots \ldots$

4.1 .2 Homomorphisms . . . . . . . . . . . . . 59

4.1.3 Natural epimorphisms and decompositions . . . . . . 61

4.2 Clans and epimorphisms . . . . . . . . . . . . . . 63

4.2.1 Homomorphism theorem . . . . . . . . . . . . 63

4.2 .2 Prime clans in quotients . . . . . . . . . . 66

4.2.3 Primitive quotients . . . . . . . . . . . . 68

4.3 Other operations . . . . . . . . . . . . . 70

4.3.1 Premorphisms . . . . . . . . . . . 70

4.3.2 Extensions . . . . . . . . . . . . . 71

Chapter 5 Clan Decomposition $\quad 75$

5.1 The clan decomposition theorem . . . . . . . . . . 75 
5.1.1 Maximal prime clans.............. 75

5.1 .2 Special sibas and 2-structures . . . . . . . . . . 77

5.1.3 The clan decomposition theorem . . . . . . . 79

5.1.4 The relationship of sibas to 2 -structures . . . . . . 81

5.2 The shape of a 2 -structure . . . . . . . . . . . . 83

5.2.1 The shape and its representation as a tree . . . . . 83

5.2 .2 Sarne shapes . . . . . . . . . . . . . 84

5.3 A construction of prime clans . . . . . . . . . . 87

5.3 .1 A construction of clans $\ldots \ldots \ldots$. . . . . 87

5.3 .2 A construction of prime clans . . . . . . . . 88

$\begin{array}{lll}\text { Chapter 6 Primitive 2-Structures } & 91\end{array}$

6.1 Small primitive substructures . . . . . . . . . . . . . 91

6.1.1 Uniformly imprimitive 2-structures . . . . . . . . 91

6.1.2 Primitive substructures of 3 or 4 nodes . . . . . . 93

6.2 Hereditary properties . . . . . . . . . . . . . . 96

6.2.1 Local and global nodes . . . . . . . . . . . 96

6.2 .2 Hereditary properties . . . . . . . . . . . . 98

6.3 Critically primitive 2 -structures . . . . . . . . . . . 99

6.3.1 The parity theorem .............. 99

6.3.2 The list of critically primitive 2 -structures . . . . . . 102

Chapter 7 Angular 2-Structures 109

7.1 Angularity . . . . . . . . . . . . . . . . . . 109

7.1.1 All-connectivity . . . . . . . . . . . . . 109

7.1.2 All-connected skew angular 2-structures . . . . . . 113

$7.2 \mathrm{~T}$-structures . . . . . . . . . . . . 116

7.2 .1 T-structures and partial orders . . . . . . 116

$7.2 .2 \quad T_{2}$-structures $\ldots \ldots \ldots \ldots \ldots \ldots \ldots \ldots$

7.3 Linear orders and Schröder nurnbers . . . . . . . . . . . 121

7.3.1 Bi-orders and linear orders . . . . . . . . . 121

7.3.2 Uniformly imprimitive linear orders . . . . . . . . 122

7.3.3 Parenthesis words and Schröder numbers . . . . . . 124

Chapter 8 Labelled 2-Structures 129

8.1 Introduction to $\ell 2$-structures $\ldots \ldots \ldots \ldots \ldots$

8.1 Definitions . . . . . . . . . . . . . 129

8.1 .2 Substructures, clans and quotients . . . . . . . 132 
8.2 Clan decomposition of $\ell 2$-structures $\ldots \ldots \ldots \ldots \ldots$

8.2.1 Uniqueness of decompositions . . . . . . . . . 135

8.2.2 The shape of an $\ell 2$-structure . . . . . . . . . 138

8.2 .3 Extensions . . . . . . . . . . . . . . . . . . 139

8.3 Graphs and their representations . . . . . . . . . . . 142

8.3.1 Graphs as $\ell 2$-structures . . . . . . . . . . . . . 142

8.3 .2 On cornparability graphs ............. 143

Chapter 9 Unstable Labelled 2-Structures 147

9.1 Triangle free and unstable $\ell 2$-structures . . . . . . . . . . 147

9.1.1 Removable edges . . . . . . . . . . . . . 147

9.1.2 Internal and external nodes . . . . . . . . . . 150

9.1.3 Triangle-free $\ell 2$-structures . . . . . . . . . . . 151

9.2 Heredity in unstable $\ell 2$-structures . . . . . . . . 155

9.2.1 The partition of nodes . . . . . . . . . 155

9.2 .2 Alternating structures $\ldots \ldots \ldots \ldots \ldots$

9.2.3 Degrees of nodes . . . . . . . . . . . . 157

9.3 A composition of unstable $\ell 2$-structures . . . . . . . . 159

9.3.1 A constructive reduction of primitive $\ell 2$-structures . . 159

9.3.2 Pendant components . . . . . . . . . . . . . 161

Chapter 10 Automorphisms of Labelled 2-Structures 165

10.1 Label preserving automorphisms . . . . . . . . . . . 165

10.1.1 The $\ell$-automorphism groups . . . . . . . . . 165

10.1.2 Transitivity . . . . . . . . . . . . . 167

10.1.3 Automorphic actions on factors . . . . . . . . 169

10.1.4 Universality of $\ell$-automorphism groups . . . . . . 172

10.2 Nonpreserving automorphisms . . . . . . . . . 175

10.2.1 Connections to $\ell$-automorphisms . . . . . . 175

10.2.2 Transitivity and associated permutations ...... 176

10.2.3 Representing labels by automorphisms . . . . . . . . 178

Chapter 11 Switching of Graphs 181

11.1 Introduction to switching . . . . . . . . . . . . 181

11.1.1 Definitions . . . . . . . . . . . . 181

11.1.2 The group of graphs . . . . . . . . . . 183

11.1.3 Switching classes . . . . . . . . . . . 185

11.2 Structural properties of switching classes . . . . . . . 188 
11.2.1 A local characterization . . . . . . . . . . 188

11.2.2 Automorphisms. . . . . . . . . . . . . . 190

11.3 Special problems on undirected graphs . . . . . . . . . 193

11.3.1 Two-graphs . . . . . . . . . . . . . . 193

11.3.2 Eulerian graphs. . . . . . . . . . . . . . . . . . 194

11.3.3 Pancyclic graphs . . . . . . . . . . . . . . . 195

11.3 .4 Trees . . . . . . . . . . . . . . . 197

Chapter 12 Labelled Structures over Groups 203

12.1 Introduction. . . . . . . . . . . . . . . . . . 203

12.1.1 Groups and involutions . . . . . . . . . . . . 203

12.1.2 Selectors and switching classes . . . . . . . . 205

12.2 An interpretation in networks . . . . . . . . . . . 207

12.2.1 Concurrent behaviour in networks . . . . . . . 207

12.2.2 Reducing the actions to groups . . . . . . . . 208

12.2.3 Introducing reversibility . . . . . . . . . . 210

12.3 Examples for some special groups . . . . . . . . . . . . 211

12.3.1 The cyclic groups $\mathbb{Z}_{3}$ and $\mathbb{Z}_{4} \ldots \ldots \ldots \ldots \ldots$

12.3.2 The symmetric group $S_{3} \ldots \ldots \ldots \ldots \ldots$

Chapter 13 Clans of Switching Classes 215

13.1 Associated groups . . . . . . . . . . . . . . 215

13.1.1 The group of selectors . . . . . . . . . . . 215

13.1.2 The group of abelian switching classes . . . . . . . 217

13.2 Clans and horizons . . . . . . . . . . . . . . 219

13.2.1 Spanning trees . . . . . . . . . . . . . . . 219

13.2.2 Horizons and constant selectors . . . . . . . . . . 220

13.2.3 Clans . . . . . . . . . . . . . . . . . 222

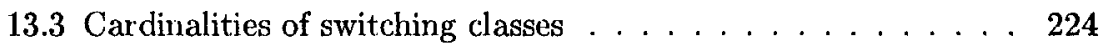

13.3.1 Sorne special cases . . . . . . . . . . . . . . 224

13.3.2 Centralizers. . . . . . . . . . . . . . . . . 224

13.3.3 Some improvements . . . . . . . . . . . . 228

Chapter 14 Quotients and Plane Trees 231

14.1 Quotients of switching classes . . . . . . . . . . . 231

14.1.1 Closure properties of clans . . . . . . . . . 231

14.1 .2 Quotients . . . . . . . . . . . . . . 233

14.2 Planes and plane trees . . . . . . . . . . . . . . 234 
14.2.1 Planes . . . . . . . . . . . . . . . 234

14.2.2 Plane trees . . . . . . . . . . . . . . . 237

14.2.3 Bijective correspondence of plane trees . . . . . . . . 239

14.2 .4 Forms . . . . . . . . . . . . . . . . 243

Chapter 15 Invariants $\quad \mathbf{2 4 5}$

15.1 Free invariants . . . . . . . . . . . . . . . . . 245

15.1.1 General invariants . . . . . . . . . . . 245

15.1.2 Edge monoids . . . . . . . . . . . . . . . . 246

15.1.3 Variable functions and free invariants . . . . . . . 247

15.2 Group properties of free invariants . . . . . . . . . . 250

15.2.1 Abelian property . . . . . . . . . . . 250

15.2.2 Graphs of words . . . . . . . . . . . . 252

15.2.3 Verbal identities . . . . . . . . . . . 255

15.3 Invariants on abelian groups . . . . . . . . . . . . . 258

15.3.1 Independency of free invariants . . . . . . . . 258

15.3.2 Complete sets of invariants . . . . . . . . . 259

15.4 Invariants on nonabelian groups $\ldots \ldots \ldots$. . . . . . . 261

15.4.1 General observations . . . . . . . . . . . . . 261

15.4.2 Central characters . . . . . . . . . . . . . 262

15.4.3 A characterization theorem . . . . . . . . . 265

$\begin{array}{ll}\text { Bibliography } & 269\end{array}$

$\begin{array}{ll}\text { Notations } & 279\end{array}$

Index 283 